\title{
Demand Response Management in Power Systems Using Particle
}

\section{Swarm Optimization}

\author{
Pedro Faria, Zita Vale, João Soares, and Judite Ferreira
}

Competitive electricity markets have arisen as a result of power-sector restructuration and power-system deregulation. The players participating in competitive electricity markets must define strategies and make decisions using all the available information and business opportunities. ${ }^{1-3}$

Demand response (DR) has proven an effective approach for allocating electrical loads, gaining a competitive advantage, and providing significant benefits for electricity market performance. DR programs can increase power consumption efficiency through active consumer participation, showing the value that each consumer attributes to his or her individualized additional demands.

Recent efforts aim to improve wholesale markets with more intensive use of DR. This includes, for example, the acceptance of demand bids and offers for ancillary services; the specification by the DR resources of the frequency, duration, and level of participation in consumption reduction; and the existence of aggregators that bid into the market on behalf of customers. ${ }^{4}$

Fully leveraging all of the advantages of active consumer participation requires an infrastructure able to accommodate all centralized and distributed energy resources. This approach corresponds to the practical implementation of smart grids, ${ }^{5}$ which are currently the focus of significant research efforts. In practice, DR programs implementation is in an initial stage, using technologies close to smart metering.

The available DR opportunities should be used in the best way to attain the involved agents' goals. This entails time-consuming and complex optimization problems, requiring huge computational means. Traditional optimization methodologies are usually not able to cope with this type of problem for realistic cases. Researchers have used artificial intelligence techniques to address several problems in the scope of power systems and electricity markets. ${ }^{6-7}$ Particle swarm optimization (PSO) ${ }^{8-9}$ has been successfully applied to power systems ${ }^{10}$ and is proposed in this work to address DR management.

This article presents a DR simulator (called DemSi) that we developed to simulate the use of DR programs. DemSi uses Power Systems CAD (PSCAD; see https:// hvdc.ca/pscad) for network simulation and 
provides users with optimized DR action management. The proposed methodology considers DR in terms of electricity price variation imposed by the distribution network operator in the presence of a reduction need for any reason, such as lack of generation or high market prices. The proposed method is based on the use of real-time pricing. This price-responsive approach would help address the difficulties in monthly fixed-remuneration programs, such as direct load control, and when the smart grid is at an initial implementation stage.

\section{Demand Response Concepts and Programs}

DR includes all intentional electricityconsumption pattern modifications by end users that alter timing, instantaneous-demand level, or total electricity consumption ${ }^{11}$ in response to changes in electricity's price over time. Further, we can also define DR as incentive payments designed to induce lower electricity use at times of high wholesale market prices or when system reliability is jeopardized. ${ }^{12}$

\section{DR Benefits}

An important advantage of DR implementation is the postponement of investments in generation resources and transmission and distribution lines. This is highly important when the generation is near its maximum capacity with exponentially increasing generation costs. In these conditions, a small reduction in load will cause a big reduction in generation costs and, therefore, a significant reduction in the price of electricity.

Usually the actions that result from demand-side behavior or those intended to manage consumer behavior are referred to as DR, load management, and demand-side management. Traditionally, these measures are taken to encourage consumers to reduce their electricity consumption during times of especially high demand ${ }^{13}$ and usually occur through utility-loadmanagement programs aiming essentially at obtaining peak reduction. Competitive electricity markets enable a wide set of new opportunities for more strategic consumer behavior and new models of DR.

Several studies have shown that loads aren't rigid, exhibiting elasticity that can be used for the mutual benefit of power systems and consumers. Changes in electricity prices over time and incentive payments increase demand flexibility as users intentionally modify their electricity consumption patterns in response to exterior stimulus. DR can be contracted over longer or shorter periods, either as a result of its inclusion in capacity markets or directly through bilateral contracts.

DR, including real-time pricing, can be used to optimize distributionnetwork operation, reduce incident consequences, and reduce wind curtailment. ${ }^{14}$ DR programs can be used to both increase and decrease load demand. The use of DR to reduce load consumption in peak, congested, and/or incident periods helps distribution network operators by relieving the network components. In this way, important benefits can be achieved in terms of reliability and service quality, cost minimization, and savings on network investments. In the case of incidents, the contracted load curtailment is expected to minimize the nonsupplied load's monetary global value. Increases of wind-based electricity generation systems and the wind's intermittent nature often lead to periods of excess generated energy. This imposes relevant losses in wind curtailment, making wind farms less efficient and the corresponding investment payback period higher. The use of DR programs to increase load consumption in these periods helps to overcome this problem.

\section{Measures and Programs}

Price elasticity is a measure used in economics to evaluate the responsiveness of the demanded quantity of a good or service to a change in its price, or the percentage change in quantity demanded in response to a one percent price change. ${ }^{15}$ In electric loads, price elasticity is a normalized measure of the intensity of how usage of electricity changes when its price changes by one percent. In the opposite way, demand elasticity is a measure of how price changes when usage of electricity changes.

Demand price elasticity can be evaluated using Equation 1, where Quantity is the quantity of the usage of the good or service and Price is the price of this good or service. ${ }^{16}$ $\Delta$ Quantity and $\Delta$ Price refer to the quantity of usage and price variations between the periods before and after the implementation of DR programs.

$$
\varepsilon=\frac{\Delta \text { Quantity } / \text { Quantity }}{\Delta \text { Price } / \text { Price }}
$$

We can divide DR programs into two general groups: price and incentive based. ${ }^{17}$ Price-based DR is related to changes in energy consumption by customers in response to variation in the prices they pay. This group includes three key pricing rates:

- Time-of-use pricing includes different prices for usage during different periods, usually defined for periods of 24 hours. This rate reflects the average cost of generating and delivering power during each period.

- Real-time pricing defines the price of electricity for shorter time periods, usually one hour, reflecting changes in the wholesale price of 
electricity. Customers usually have the information of prices one day or one hour beforehand.

- Critical-peak pricing is a hybrid of the other two pricing programs. The base program is time-of-use, with a much higher peak pricing applied under specified conditions (for example, when system reliability is compromised or supply costs are high).

For different hours or time periods, if the price varies significantly, customers can respond with changes in energy use. Response to price-based DR programs is entirely voluntary.

Incentive-based DR includes programs that give customers fixed or time-varying incentives and that complement their electricity rate. Utility companies, load-serving entities, or a regional grid operator can establish these approaches. Some of these programs penalize customers who fail the contractual response when a priori specified events are declared. This group includes programs such as direct load control, interruptible/curtailable service, demand bidding/buyback, emergency DR, capacity market, and ancillary services market.

In other work, Lorna Greening exposes the expected responsibilities and functions of each player in a deregulated electricity market. ${ }^{18}$ Greening states that larger participation is required for DR to be viable in the scope of electricity markets, and that this requires a more intensive collaboration between regulators, market participants, and market and system operators.

Katherine Hamilton and Neel Gulhar propose a complex-bid, marketclearing mechanism that considers price-sensitive bids made by consumers. ${ }^{19}$ This work quantifies the effect of the increasing participation of the demand-side on various categories of market participants. The authors conclude that the increase of demand shifting causes the reduction of marketclearing prices, benefiting all bidders even if they don't participate in the shifting activities.

Direct load control is a DR model for which the utility is able to control customer equipment. This control has shown positive results, avoiding the use of additional generation. Implemented models have been applied to air conditioners and water heaters. However, some problems related to the functioning of switches installed by the utility have been reported. In the future, with further implementation of the smart grid concept with two-way communication ability, it's expected that utilities will have better control over the target equipment. This is important because incentive payments are usually paid monthly to customers as fixed rates. ${ }^{20}$ Although this concept isn't presently considered useful, utilities are planning to offer programs in which they give customers price signals through smart pricing programs. These signals are expected to give incentives to customers to make their own investments in DR equipment. ${ }^{20}$

\section{Problem Formulation and Resolution}

The DR problem that we consider here is motivated by a need to reduce the energy supply to a set of consumers by a specified amount. This event is managed by a consumer aggregator, aiming at minimizing the global value paid by the consumers. To achieve this goal, the aggregator considers the individual consumers' price elasticity, which relates demand reduction with price increase. Individual load reduction and price increases that minimize the consumers' global cost are determined for each consumer.

\section{Problem Formulation}

The problem we aim to solve consists of the optimal minimization of the global costs from the point of view of electricity consumers, regarding loads managed by a load aggregator. The problem's characteristics lead to a nonlinear model.

In practice, when a reduction in electricity consumption is needed, the aggregator, based on its knowledge about the consumers, raises the price of electricity with the expectation that consumers will reduce their electricity use. The objective function can be expressed as shown in Equation 2 and is subjected to several constraints.

$$
\begin{aligned}
& \text { Min Cost } \\
& \sum_{c=1}^{n c\lceil} \mid \begin{array}{l}
\left(E_{\text {Load }(c)}-E_{\text {LoadRed }(c)}\right) \\
\left.\times\left(\text { Price }_{\text {EnergyInitial }(c)}+\text { Price }_{\text {Energy Var }(c)}\right)\right\rfloor
\end{array}
\end{aligned}
$$

Here, Cost is the total load consumption cost, $n c$ is the number of consumers, $E_{\text {Load (c) }}$ is initial energy consumption of consumer $c, E_{\text {LoadRed(c) }}$ is energy consumption reduction of consumer $c$, Price $_{\text {EnergyInitial(c) }}$ is initial electricity price for consumer $c$, and Price EnergyVar(c) $_{\text {is variation in con- }}$ sumer $c$ electricity price. The objective function in Equation 2 aims to minimize costs associated with electricity consumption (that is, the total amount consumers pay) when an overall demand reduction is required. We can calculate these costs based on the final load demand (initial load demand minus demand reduction value) and on the final price (initial price plus the price increment used to obtain the required consumption reduction).

Limitations are imposed on each customer's power (see Equation 3) and price (Equation 4) variations 
according to the extent in which they can and/or want to participate in the DR program and to their price elasticity. Power system operation requires the balance between load and generation to be guaranteed at all times (see Equation 5).

$P_{\text {LoadRed(c) }} \leq \operatorname{Max}_{\text {LoadRed(c) }}$

Price $_{\text {EnergyVar(c) }} \leq$ MaxPrice $_{\text {EnergyVar(c) }}$

$P_{\text {Main }}-P_{\text {Reserve }}=\sum_{c=1}^{n c} P_{\text {Load }(c)}-\sum_{c=1}^{n c} P_{\text {LoadRed }(c)}$,

where $\operatorname{Max} P_{\text {LoadRed(c) }}$ is the maximum permitted variation in power for consumer $c$; MaxPrice $_{\text {EnergyVar(c) }}$ is the maximum permitted variation in energy price for consumer $c$; $P_{\text {Main }}$ is power received from the main grid; $P_{\text {Reserve }}$ is reserve power; $P_{\text {Load (c) }}$ is initial power consumption of consumer $c$; and $P_{\text {LoadRed(c) }}$ is power consumption reduction of consumer $c$.

The consideration of load response is formulated based on elasticity values (see Equation 6). Because the elasticity is a fixed and constant value for each load, the optimal relation between load and price variation is determined in the optimization. The present study considers the obligation of having the same price variation for the loads of the same type as expressed in Equation 7.

$$
=\frac{P_{\text {LoadRed }(c)} \times \text { Price }_{\text {EnergyInitial }(c)}}{P_{\text {Load }(c)} \times \text { Price }_{\text {EnergyVar }(c)}}
$$

$$
\begin{aligned}
& \text { Price }_{\text {EnergyVar(c) }} \\
& =\text { Price }_{\text {EnergyVar(T) }}, \quad \forall c \in T,
\end{aligned}
$$

where Elasticity $_{(c)}$ is price elasticity for consumer $c$, and $T$ is consumer type.

\section{Particle swarm Optimization}

The optimization of the formulated nonlinear problem consists of the minimization of a multimodal function with many local minima and a global optimum. This is considered an NP-hard problem because the computational complexity is high even in simple cases.

In past decades, AI techniques have deployed a set of effective and efficient methods to mitigate the difficulties of solving complex computational time problems. These algorithms explore a given search space and return the best solution found. PSO belongs to the category of swarm intelligence methods, and we use it in this work to solve the DR problem because it's effective in difficult optimization tasks such as nonlinear problems. ${ }^{21}$ We describe the algorithm as follows:

\section{START}

2. Initialization of parameters (maximum velocities, minimum velocities, position limits, maximum iterations)

3. Random generation of initial values (swarm)

4. REPEAT

5. Reproduction: Each particle generates 1 new descendent (movement, new position)

6. Evaluation: Each particle has its fitness value, according to its current position in search space

7. Store the best solution of swarm

8. UNTIL termination criteria (Number of generations)

9. END PSO

We then compared the results and performance of this technique with those obtained with conventional techniques using the professional optimization tool, General Algebraic Modeling System (GAMS; see www. gams.com).

\section{Simulator}

DemSi is a DR simulator that we developed to simulate the use of DR programs. We used PSCAD as the base platform for the network simulation, enabling the use of detailed models of electrical equipment and the consideration of transient phenomena. These abilities are relevant to analyze the technical viability of the DR proposed solutions, both for steady state and transients (although we don't present network response to load changes here).

DemSi considers the players involved in the DR actions and allows result analysis from each specific player's viewpoint. This includes four types of players: electricity consumers, consumer aggregators, electricity retailers (suppliers), and the distribution network operator. Here, we analyze the case study from the viewpoint of a consumers' aggregator.

Consumers can be characterized on an individual or aggregated basis. Based on their profiles, some clients can establish flexible supply contracts with their suppliers. DemSi considers the information concerning the quantity of load that can be cut or reduced and the corresponding compensations for each client.

DemSi classifies the loads as five main types based on function of peak power consumption, energy destination, and load diagram:

- domestic,

- small commerce,

- medium commerce,

- large commerce, and

- industrial.

Figure 1 shows DemSi's general architecture.

To fully attain our goals, PSCAD is linked with Matlab (see www. mathworks.com) and GAMS. These links let us use programmed modules 
to model the relevant players' behavior and relationships, focusing on the contracts between each client and each supplier. The formulated optimization problem's solution is found using Matlab and/or GAMS. Using diverse approaches for solving the optimization problems, it's possible to derive the best approach for each type of situation.

Once the simulation is started, a simulation timeline feeds DemSi with timetagged events. PSCAD simulates all physical phenomena related to the power system. The management of DR programs is undertaken by a module developed in Matlab. The simulation's end is determined by the end of the simulation timeline.

Every time the simulator is initiated, an initial state (for example, load value, breaker state, and so on) is considered as the departing simulation point. Once the simulation is launched, the supply information and the consumer knowledge base have the required information that allows optimizing DR program use over time, allowing the simulation to continue. The DR program-management module optimizes the use of DR opportunities for each situation.

\section{Case Studies}

Here, we illustrate the use of the proposed methodology in the developed DR simulator DemSi. The case study considers a distribution network with 32 buses from Mesut Baran and Felix $\mathrm{Wu},{ }^{22}$ which we evolved in a scenario to the year 2,040 in terms of load characterization. ${ }^{23}$ All the results presented in this article are obtained for two scenarios, with 32 and 320 consumers, respectively.

\section{Case Characterization}

Table 1 shows the load demand in the first scenario, with 32 consumers. The second scenario had 10 consumers in each bus, corresponding to a total of 320 consumers. In this scenario, the 10 loads connected to each bus have the total power presented in Table 1 and are of the same load type. Table 1 also shows each consumer's type.

In both scenarios, we considered that all loads of the same type have the same price variation during the DR program application. We solved each scenario and reduction need with two approaches: with the developed PSO module and with nonlinear programming (NLP) implemented in GAMS. We compared results in terms of time of execution and solution values. The values of elasticity are 0.14 ,
$0.12,0.20,0.28$, and 0.38 , respectively, for domestic, small commerce, medium commerce, large commerce, and industrial consumer types. The corresponding values for electricity price, which correspond to retailer's flat-rate tariff values, are $0.18,0.19,0.20$, 1.16 , and 0.12 euros per kW hour.

As a restriction on the proposed formulation, we consider a price and power cap; we can parameterize these cap values for each case study. For this case study, the price cap is equal to 150 percent of the energy price value and the power cap is 15 percent of the power consumption value for every customer.

DR program use is triggered by a load reduction required by the supplier. We consider a set of seven reduction values for each scenario. For each reduction requirement, the energy price for each consumer type and the load reduction for each consumer are obtained as a result of the optimization problem.

\section{Particle swarm Optimization application Details}

The problem described here has five variables that are the price variation upper limits for the five load types. In PSO, these variables are easily coded-that is, each particle has a dimension space of five. Table 2 shows the results of the performance and parameters sensitivity analysis of the method for 1,000 runs.

Given our results, we adopted configuration A for this case study. The maximum position of each particle's 
Table 1. Consumer data.

\begin{tabular}{|c|c|c|}
\hline Bus & Power (kW) & Consumer type \\
\hline 1 & 169.1 & Medium commerce \\
\hline 2 & 148.9 & Small commerce \\
\hline 3 & 147.1 & Small commerce \\
\hline 4 & 145.5 & Small commerce \\
\hline 5 & 94.2 & Domestic \\
\hline 6 & 311.1 & Large commerce \\
\hline 7 & 308.7 & Large commerce \\
\hline 8 & 89.3 & Domestic \\
\hline 9 & 90.6 & Domestic \\
\hline 10 & 67.0 & Domestic \\
\hline 11 & 91.1 & Domestic \\
\hline 12 & 91.3 & Domestic \\
\hline 13 & 181.3 & Medium commerce \\
\hline 14 & 91.1 & Domestic \\
\hline 15 & 91.1 & Domestic \\
\hline 16 & 91.9 & Domestic \\
\hline 17 & 135.5 & Small commerce \\
\hline 18 & 152.4 & Medium commerce \\
\hline 19 & 151.7 & Medium commerce \\
\hline 20 & 151.6 & Medium commerce \\
\hline 21 & 151.5 & Medium commerce \\
\hline 22 & 147.3 & Small commerce \\
\hline 23 & 674.8 & Industrial \\
\hline 24 & 669.3 & Industrial \\
\hline 25 & 93.8 & Domestic \\
\hline 26 & 93.2 & Domestic \\
\hline 27 & 92.2 & Domestic \\
\hline 28 & 183.0 & Medium commerce \\
\hline 29 & 295.3 & Medium commerce \\
\hline 30 & 225.4 & Medium commerce \\
\hline 31 & 315.1 & Large commerce \\
\hline 32 & 89.8 & Domestic \\
\hline Total & $5,831.3$ & - \\
\hline
\end{tabular}

dimension is the price variation upper limit; the minimum position is zero. This case study uses 60 particles and 200 iterations. The maximum and minimum velocity are 0.01 and -0.1 , respectively.

We used control penalties to address the energy balance constraint.
We added the penalties to the fitness function to control the system energy balance (equality equation).

\section{Results}

We analyze the results in terms of execution time function and the obtained solutions. We verified that ex- ecution time doesn't depend on the consumption reduction need. PSO takes about 0.07 and 0.15 seconds for 32 and 320 consumers, respectively. NLP takes 0.32 seconds in both cases.

Although the execution time difference between PSO and NLP might seem irrelevant, the advantage of the PSO approach is important for some studies for which a large number of simulations is required in a relatively short time. This could be the case when deciding to declare a DR event when the system has a minimum anticipation time for consumer notification.

Figure 2 shows the values of each approach's objective function and each scenario's reduction need. These values, in euros, correspond to the global costs for the loads after the DR program implementation.

Comparing PSO and NLP, we can conclude that, for lower reduction needs, the results are almost the same. For higher reduction needs, PSO returns slightly worse results. These conclusions are not influenced by the problem's dimension (that is, the number of consumers).

For a more detailed analysis, Figure 3 shows the energy price variation for the 32 loads scenario for each approach. In Figure 3a, results for the major reduction need are grouped by consumer type. For this reduction need, all loads are required to participate. The slight differences between the two approaches are because PSO is a stochastic method. Because finding the global optimum can't be guaranteed, the obtained solution tends to be a local optimum with an objective function value close to the global optimum. Although the value obtained for the objective function is close to the one obtained by the NLP approach, the solution itself can present some differences-namely, 
Table 2. Particle swarm optimization parameter-sensitivity analysis.

\begin{tabular}{ccccccccc} 
Configuration & $\begin{array}{c}\text { Maximum } \\
\text { velocity }\end{array}$ & $\begin{array}{c}\text { Minimum } \\
\text { velocity }\end{array}$ & $\begin{array}{c}\text { No. of } \\
\text { iterations }\end{array}$ & $\begin{array}{c}\text { Solutions } \\
\text { without } \\
\text { violations (\%) }\end{array}$ & Mean fitness & $\begin{array}{c}\text { Worst } \\
\text { fitness }\end{array}$ & $\begin{array}{c}\text { Best } \\
\text { fitness }\end{array}$ & $\begin{array}{c}\text { Meantime } \\
\text { (s) }\end{array}$ \\
\hline A & 0.01 & -0.1 & 200 & 94 & $1,422.20$ & $1,482.00$ & $1,379.80$ & 0.0716 \\
B & 0.01 & -0.01 & 200 & 93 & $1,427.00$ & $1,534.00$ & $1,377.50$ & 0.0715 \\
\hline C & 0.01 & -0.1 & 100 & 82 & $1,426.60$ & $1,527.10$ & $1,366.70$ & 0.0387 \\
D & 0.01 & -0.01 & 100 & 82 & $1,433.10$ & $1,654.70$ & $1,378.50$ & 0.0382 \\
\hline
\end{tabular}

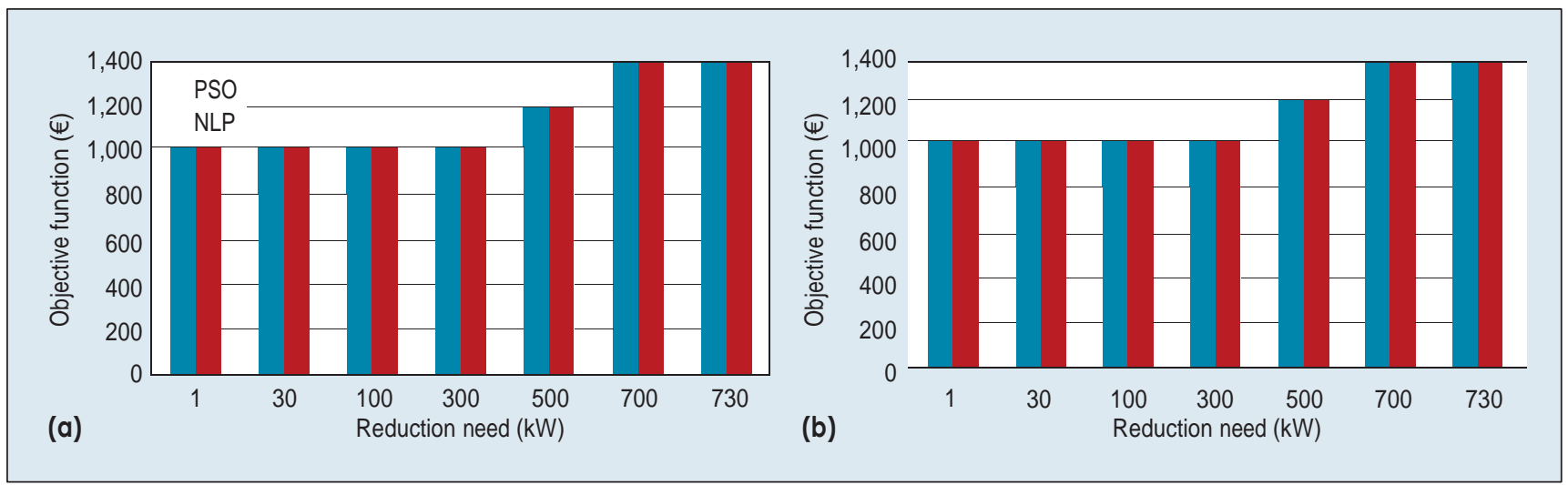

Figure 2. The global costs for loads after DR implementation. The costs are determined by each approach's objective function and each scenario's reduction needs, which are shown here for (a) 32 loads and (b) 320 loads. For lower reduction needs, the results are almost the same. For higher reduction needs, PSO returns slightly worse results.
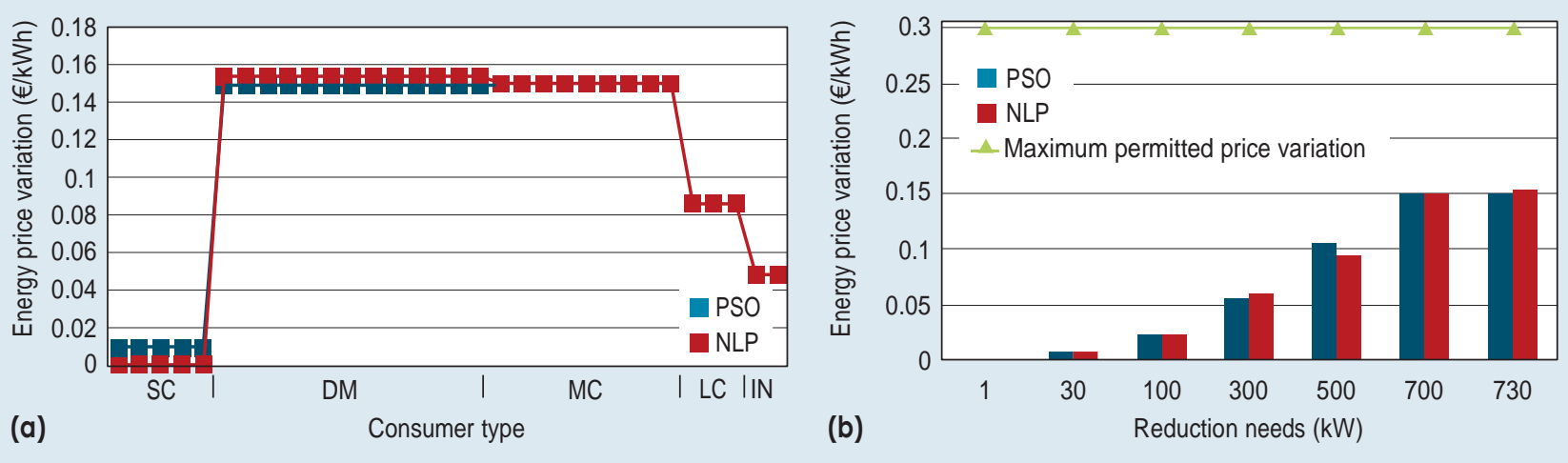

Figure 3. Detailed results for energy price variation for the 32 loads scenario. (a) Price variation in the major reduction need for each consumer, grouped by consumer type. (b) Major energy price variation in comparison with the maximum permitted.

in what concerns the consumers involved in the load reduction for each specific DR event. In Figure $3 b$, for each reduction need, the maximum variation on energy price is compared with the maximum allowed variation. In all results, the variations are largely below the maximum permitted because the formulation considers both price and power cap, and the power cap became prevalent, and hence it limited higher response from loads.

Finally, Figure 4 shows the DR program's load participation in the two scenarios. In general, comparing the results for the two scenarios shows similar behavior. In terms of the number of loads affected, the PSO solutions tend to correspond to more distributed load participation. For the loads that reached the maximum power variation, NLP performs rational management of loads, scheduling loads like using an order of merit: the next load is used when the load under consideration has no more capacity. In contrast, PSO spreads the variation among the loads so the maximum variation is not reached except for higher reduction needs when load variations are forced to the limit. 


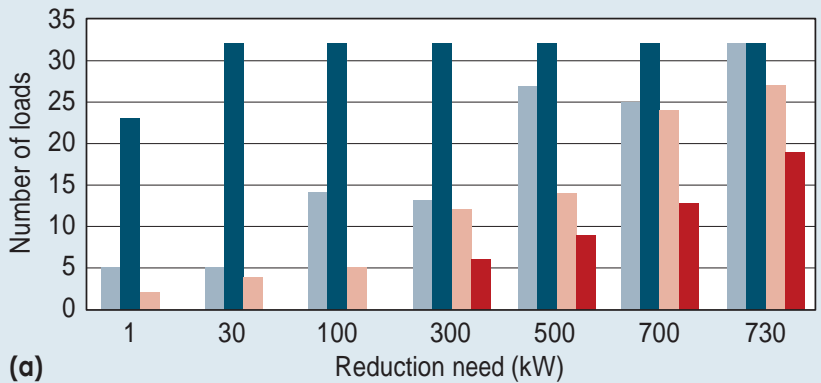

NLP - Number of affected loads

NLP - Number of loads that reached maximum load variation

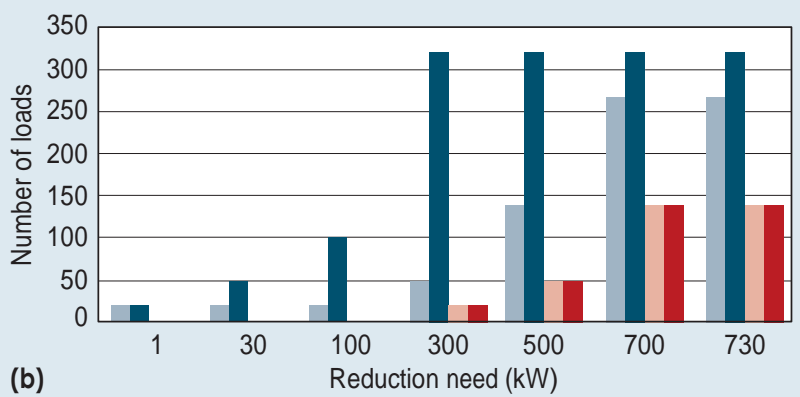

PSO - Number of affected loads

PSO - Number of loads that reached maximum load variation

Figure 4. The DR program's load participation. This shows both the number of loads that have participated and the loads that reached maximum load variation in terms of reduction need for the (a) 32 load and (b) 320 load scenarios.

In terms of the number of loads affected by DR, the PSO solution gives higher load participation for each imposed consumption reduction. PSO tends not to achieve the maximum load reduction for each consumer, spreading load reduction by more load types. For these reasons, the PSO approach leads to more interesting solutions, achieving the envisaged consumption needs involving a large number of participants, with only a slight increase of the total cost.

\section{Acknowledgments}

This work is supported by Fundo Económico para o Desenvolvimento Regional (FEDER) funds through the Fatores de Competitividade (COMPETE) program and by national funds through Fundação para a Ciência e a Tecnologia (FCT) under the projects FCOMP-01-0124-FEDER: PEst-OE/ EEI/UI0760/2011, PTDC/EEA-EEL/099832/ 2008, PTDC/SEN-ENR/099844/2008, and PTDC/SEN-ENR/122174/2010.

\section{References}

1. Z. Vale et al., "Mascem-Electricity Markets Simulation with Strategically Acting Players," IEEE Intelligent Systems, vol. 26, no. 2, 2011, pp. 9-17.

2. Y. Guan and M. Kezunovic, "Grid Monitoring and Market Risk Management," IEEE Intelligent Systems, vol. 26, no. 2, 2011, pp. 18-21.

3. J. Ferreira et al., "A Data-MiningBased Methodology for Transmission
Expansion Planning," IEEE Intelligent Systems, vol. 26, no. 2, 2011,

pp. 28-37.

4. F. Rahimi and A. Ipakchi, “Overview of Demand Response under the Smart Grid and Market Paradigms," Proc. Innovative Smart Grid Technologies, IEEE, 2010; doi:10.1109/ ISGT.2010.5434754.

5. Z. Vale, H. Morais, and H. Khodr, "Intelligent Multi-Player Smart Grid Management Considering Distributed Energy Resources and Demand Response," Proc. IEEE Power and Energy Society General Meeting, IEEE, 2010, pp. 1, 7, 25-29; doi:10.1109/ PES.2010.5590170.

6. K.Y. Lee and M.A. El-Sharkawi, Modern Heuristic Optimization Techniques: Theory and Applications to Power Systems, Wiley-Interscience, 2008.

7. C. Ramos and C. Liu, "Intelligent Systems in Power Systems and Energy Markets," IEEE Intelligent Systems, vol. 26, no. 2, 2011, pp. 5-8.

8. J. Kennedy and R.C. Eberhart, "Particle Swarm Optimization," Proc. IEEE Int'l Conf. Neural Networks, vol. 4, IEEE, 1995, pp. 1942-1948.

9. E. Bonabeau, M. Dorigo, and G. Theraulaz, Swarm Intelligence: From Natural to Artificial Systems, Oxford Univ. Press, 1999.

10. M.R. Al-Rashidi, and M.E. El-Hawary, "A Survey of Particle Swarm Optimization Applications in Electric Power
Systems," IEEE Trans. Evolutionary Computation, vol. 13, no. 4, 2009, pp. 913-918.

11. D.S. Kirschen, "Demand-Side View of Electricity Markets," IEEE Trans. Power Systems, vol. 18, no. 2, 2003, pp. 520-527.

12. P.R. Thimmapuram et al., "Modeling and Simulation of Price Elasticity of Demand Using an Agent-Based Model," Proc. Innovative Smart Grid Technologies, IEEE, 2010.

13. L. Stuntz, Keeping the Lights On in a New World, tech. report, US DOE Electricity Advisory Committee, Jan. 2009.

14. Z. Vale et al., "The Role of Demand Response in Fuvture Power Systems," Proc. Transmission and Distribution Conf. \& Exposition: Asia and Pacific, IEEE, 2009; doi:10.1109/ TD-ASIA.2009.5356902.

15. R.A. Arnold, Economics, 9th ed., South-Western College Pub, 2008.

16. M.H. Albadi and E.F. El-Saadany, "ASummary of Demand Response in Electricity Markets," Electric Power Systems Research, vol. 78, no. 11, 2008, pp. 1989-1996.

17. J. Bushnell, B. Hobbs, and F. Wolak, "When It Comes to Demand Response, Is FERC Its Own Worst Enemy?" The Electricity J., vol. 22, no. 8, 2009, pp. 9-18.

18. L.A. Greening, "Demand Response Resources: Who Is Responsible for Implementation in a Deregulated 
Market?" Energy, vol. 35, no. 4, 2010, pp. 1518-1525.

19. K. Hamilton and N. Gulhar, "Taking Demand Response to the Next Level," IEEE Power and Energy, vol. 8, no. 3, 2010, pp. 60-65.

20. S. Chua-Liang and D. Kirschen, "Quantifying the Effect of Demand Response on Electricity Markets," IEEE Trans. Power Systems, vol. 24, no. 3, 2009, pp. 1199-1207.

21. A. Engelbrecht, Computational Intelligence: An Introduction, John Wiley \& Sons, 2007.

22. M. Baran and F. Wu, "Network Reconfiguration in Distribution Systems for Loss Reduction and Load Balancing," IEEE Trans. Power Delivery, vol. 4, no. 2, 1989, pp.1401-1407.

23. P. Faria, Z. Vale, and J. Ferreira, "DemSi-A Demand Response Simulator in the Context of Intensive 